\title{
Pooled Safety Results Through 1 Year of 2 Phase III Trials of Guselkumab in Patients With Psoriatic Arthritis
}

\author{
Proton Rahman ${ }^{1}$, Christopher T. Ritchlin' ${ }^{2}$ Philip S. Helliwell ${ }^{3}$ (D), Wolf-Henning Boehncke ${ }^{4}$, \\ Philip J. Mease ${ }^{5}$ (D), Alice B. Gottlieb ${ }^{6}$ (D), Shelly Kafka ${ }^{7}$, Alexa P. Kollmeier ${ }^{8}$, Elizabeth C. Hsia ${ }^{9}$, \\ Xie L. Xu ${ }^{8}$, May Shawi ${ }^{10}$, Shihong Sheng ${ }^{11}$, Prasheen Agarwal ${ }^{11}$, Bei Zhou ${ }^{11}$, Paraneedharan Ramachandran ${ }^{11}$, \\ Yanli Zhuang ${ }^{11}$, and Iain B. McInnes ${ }^{12}$ (D)
}

\begin{abstract}
Objective. Evaluate the safety of guselkumab (monoclonal antibody targeting interleukin [IL]-23p19) in patients with psoriatic arthritis (PsA) through 1 year (1Y) of the phase III DISCOVER-1 and DISCOVER-2 trials.

Methods. Patients with active PsA ( $\mathrm{n}=1120$; biologic-naïve except 118 patients treated with tumor necrosis factor inhibitors in DISCOVER-1) were randomized to subcutaneous guselkumab $100 \mathrm{mg}$ every 4 weeks (Q4W) or at Week 0, Week 4, then every 8 weeks (Q8W); or placebo. At Week 24, patients in the placebo group switched to guselkumab $100 \mathrm{mg}$ Q4W. Treatment continued through $1 \mathrm{Y}$ and 2 years for DISCOVER-1 and DISCOVER-2, respectively. In this pooled analysis, patients with $\geq 1$ adverse event (AE) through $1 Y$ were standardized for 100 patient-years [100 PYs] of follow-up.

Results. Through Week 24, adverse events (AEs) were consistent between patients treated with placebo and guselkumab (Q4W + Q8W). AEs were 142.8/100 PYs and 150.6/100 PYs, serious AEs were 7.1/100 PYs and 4.4/100 PYs, and AEs leading to study agent discontinuation were 4.1/100 PYs and 3.8/100 PYs, respectively. Through $1 \mathrm{Y}$ in patients treated with guselkumab, no uveitis, active tuberculosis, opportunistic infections, or inflammatory bowel disease were observed, and low rates of malignancy and major adverse cardiovascular $(\mathrm{CV})$ events were observed. Injection-site reactions occurred in $1.7 \%$, and antibodies to guselkumab in $4.5 \%$ of patients treated with guselkumab through $1 Y$; the vast majority of antibodies to guselkumab were nonneutralizing. Serum hepatic transaminase elevations (more common with Q4W than Q8W dosing) and decreased neutrophil counts were generally mild, transient, and did not require treatment discontinuation, with minimal change from Week 24 to $1 \mathrm{Y}$.

Conclusion. Guselkumab $100 \mathrm{mg}$ Q4W and Q8W were well tolerated in patients with PsA, with no new safety concerns through $1 Y$ of the phase III DISCOVER trials. Guselkumab safety through $1 Y$ in patients with PsA is consistent with that established in patients with psoriasis who were treated with guselkumab. [ClinicalTrials.gov: NCT03162796 and NCT03158285]
\end{abstract}

Key Indexing Terms: adverse effects, biologics, psoriatic arthritis, safety, tolerability

\footnotetext{
This study was sponsored by Janssen Research \& Development.

${ }^{I}$ P. Rahman, MD, Memorial University of Newfoundland, St. John's, Newfoundland, Canada; ${ }^{2}$ C.T. Ritchlin, $M D, M P H$, University of Rochester, Rochester, New York, USA; ${ }^{3}$ P.S. Helliwell, PhD, University of Leeds, Leeds, UK; ${ }^{4}$ W.H. Boehncke, MD, Geneva University Hospitals, Geneva, Switzerland; ${ }^{5}$ P.J. Mease, MD, Swedish Medical Center/Providence St Joseph Health and University of Washington School of Medicine, Seattle, Washington, USA ${ }^{6}$ A.B. Gottlieb, MD, PhD, Icahn Medical School at Mount Sinai, New York, New York, USA; ${ }^{7}$ S. Kafka, MD, Janssen Scientific Affairs, Horsham, Pennsylvania, USA; ${ }^{8}$ A.P. Kollmeier, MD, X.L. Xu, PhD, Janssen Research \& Development, San Diego, California, USA; ${ }^{9}$ E.C. Hsia, MD, Janssen Research \& Development, Spring House, Pennsylvania, USA, and University of Pennsylvania School of Medicine, Philadelphia, Pennsylvania, USA; ${ }^{10}$ M. Shawi, PhD, Immunology Global Medical Affairs, Janssen Pharmaceutical Companies of Johnson \& Johnson, Horsham, Pennsylvania, USA; ${ }^{11}$ S. Sheng, PhD, P. Agarwal, PhD, B. Zhou, PhD, P. Ramachandran, MD, MPH, Y. Zhuang, PhD, Janssen Research \& Development, Spring House, Pennsylvania, USA; ${ }^{12} I . B$. McInnes, MD, PhD, University of Glasgow, Glasgow, UK.

$C T R$ has received research support from AbbVie, Amgen, and UCB, and
}

consultant fees from AbbVie, Amgen, Gilead, Janssen, Lilly, Novartis, Pfizer, and UCB. PR has received research support from Janssen and Novartis; consultant fees from Abbott, AbbVie, Amgen, BMS, Celgene, Eli Lilly, Janssen, Novartis, Pfizer, Roche, and UCB; and speakers bureau support from AbbVie, Eli Lilly, Janssen, Novartis, Pfizer, and UCB. PSH has received consulting fees from Eli Lilly and fees for educational services from Amgen, Janssen, Novartis, and Pfizer. WHB has received research support from Pfizer and consulting fees and speaker bureau support from AbbVie, Almirall, Celgene, Janssen, LEO Pharma, Eli Lilly, Novartis, and UCB. IBM has received research support from AstraZeneca, BMS, Celgene, Janssen, Eli Lilly, Novartis, and UCB, and consultant fees from AbbVie, BMS, Boehringer Ingelheim, Celgene, Eli Lilly, Gilead, Janssen, Novartis, and UCB. ABG has received research support from Boehringer Ingelheim, Incyte, Janssen, Novartis, Sun Pharmaceutical Industries, UCB, and XBiotech; honoraria from Amgen, BMS, Celgene, Janssen, LEO Pharma, Novartis, and UCB Pharma; consultant fees from AnaptysBio, Avotres, Boehringer Ingelheim, Incyte, Lilly ICOS, Novartis, Pfizer, and Sun Pharmaceutical Industries; and stock options from XBiotech. PJM has received research support, consulting fees, and speaker bureau support from AbbVie, Amgen, BMS, Celgene, Crescendo Bioscience, Genentech, Janssen, Eli Lilly, Merck, Novartis, Pfizer, 
Guselkumab is a novel human monoclonal antibody that binds to the p19 subunit of interleukin (IL)-23 with high affinity. Guselkumab prevents binding of IL-23 to the IL-23 receptor and inhibits release of proinflammatory cytokines. ${ }^{1,2}$ IL-23 has been implicated in the pathogenesis of autoimmune diseases, including psoriasis ( $\mathrm{PsO}$ ), psoriatic arthritis ( $\mathrm{PsA}$ ), and inflammatory bowel disease (IBD). ${ }^{3,4,5}$ IL-23 is an important driver of Th17 cell differentiation and survival and an upstream regulator of IL-17A, a central proinflammatory effector cytokine in $\mathrm{PsO}$ pathogenesis. ${ }^{5,6,7}$ Guselkumab is the first IL-23p19-subunit inhibitor approved to treat moderate-to-severe $\mathrm{PsO}$ and active PsA. $1,8,9,10,11$

PsA is a seronegative, chronic, inflammatory arthropathy that occurs in approximately $30 \%$ of patients with PsO. ${ }^{12} \mathrm{IL}-23$ can also induce IL-22 (a cytokine important in enthesitis and excess bone formation) and elicit joint damage, in part by IL-17A and tumor necrosis factor (TNF) induction. Two phase III studies, DISCOVER-1 and DISCOVER-2, demonstrated that guselkumab is efficacious in treating the signs and symptoms of active PsA and inhibiting structural damage progression, ${ }^{9,10}$ with sustained response rates and low levels of radiographic progression seen through 1 year. ${ }^{13,14}$

As most patients with PsA who receive biologics will require continual therapy to maintain control of their disease, understanding the safety of long-term treatment with cumulative exposure, particularly for a new mechanism of action for the disease, is critical. The treatment of rheumatologic conditions with biologic agents may be associated with long-term adverse effects, most commonly serious infections, particularly with anti-TNF agents. ${ }^{15,16}$ Other less common serious adverse effects are associated with biologic therapies and may be specific to a select target. ${ }^{17}$

The long-term safety results through 4 years of 2 pivotal phase III studies, VOYAGE-1 and VOYAGE-2, of guselkumab $100 \mathrm{mg}$ every 8 weeks (Q8W) in patients with PsO have been published. ${ }^{8,11,18,19,20}$ Safety results from DISCOVER-1 and DISCOVER-2 have been reported separately through the placebo-controlled periods $s^{9,10}$ and also through 1 year. ${ }^{13,14}$ Here, we report the pooled safety results of guselkumab $100 \mathrm{mg}$ every 4 weeks $(\mathrm{Q} 4 \mathrm{~W})$ and $\mathrm{Q} 8 \mathrm{~W}$ in patients with PsA, including time-adjusted incidences of adverse events (AEs) and AEs of special interest, as well as clinical laboratory results, through 1 year of DISCOVER-1 and DISCOVER-2.

\section{METHODS}

Study design. DISCOVER-19 and DISCOVER-2 ${ }^{10}$ were randomized, double-blind, phase III trials of guselkumab in patients with active PsA

and UCB. SK was an employee of Janssen Scientific Affairs at the time this work was performed; $A P K, E C H, X L X, S S, P A, B Z, P R$, and $Y Z$ are employees of Janssen Research \& Development; and MS is an employee of Janssen Global Services - these authors own stock or stock options in Johnson \&Johnson.

Address correspondence to Dr. P. Rahman, 154 LeMarchant Road,

St. Clare's Mercy Hospital, St. John's, NL AIC 5B8, Canada.

Email:prahman@mun.ca.

Accepted for publication April 20, 2021. who had inadequate responses to standard therapies (Figure 1). Patients were randomized 1:1:1 to receive subcutaneous (SC) guselkumab $100 \mathrm{mg}$ at Week 0, then Q4W; guselkumab $100 \mathrm{mg}$ at Weeks 0, 4, then Q8W; or placebo Q4W (Figure 1). Stable doses of nonsteroidal antiinflammatory drugs (NSAIDs), oral corticosteroids, and selected nonbiologic disease-modifying antirheumatic drugs (DMARDs) were permitted. At Week 16, early escape to initiation or increase of allowed concomitant PsA medications was available to patients with $<5 \%$ improvement in both tender and swollen joint counts. At Week 24, patients receiving placebo crossed over to receive guselkumab 100 mg Q4W. In DISCOVER-1, treatment continued through Week 48 with a final follow-up safety visit at Week 60; treatment continued through Week 100 of DISCOVER-2, with a final follow-up safety visit at Week 112 (data through $1 \mathrm{yr}$ are included in these analyses). Upon premature discontinuation of treatment, patients had a final safety visit approximately 12 weeks after the last study agent administration.

The trials were conducted in accordance with the principles of the Declaration of Helsinki and Good Clinical Practices. Protocols were approved by ethics committees at each site (Sterling institutional review board approval numbers [US sites]: 5959C and 5910C), and all patients provided written informed consent. The trials are registered at ClinicalTrials. gov: NCT03162796 and NCT03158285.

Patients. Detailed inclusion and exclusion criteria for DISCOVER-1 and DISCOVER-2 have been reported. ${ }^{9,10}$ Briefly, enrolled patients were adults with active PsA despite previous therapy with DMARDs, apremilast, and/or NSAIDs. Patients had been diagnosed with PsA for at least 6 months and met the Classification Criteria for Psoriatic Arthritis. In DISCOVER-1, patients were required to have $\geq 3$ swollen joints, $\geq 3$ tender joints, and C-reactive protein $(\mathrm{CRP}) \geq 0.3 \mathrm{mg} / \mathrm{dL}$. In DISCOVER-2, patients were required to have $\geq 5$ swollen joints, $\geq 5$ tender joints, and CRP $\geq 0.6 \mathrm{mg} / \mathrm{dL}$. Patients were biologic-naïve with the exception of approximately $30 \%$ of patients in DISCOVER-1 who had previously received 1 or 2 anti-TNF agents. Exclusion criteria included other inflammatory diseases such as rheumatoid arthritis; specified infections including active tuberculosis (TB); most malignancies within 5 years of screening; and any prior use of Janus kinase inhibitors, or phototherapy or systemic immunosuppressants within 4 weeks of study agent administration.

Safety assessments. Tolerability of guselkumab (through Week 60 in DISCOVER-1 and Week 52 of DISCOVER-2) was evaluated based on reports of AEs, clinical laboratory investigations (abnormalities classified by National Cancer Institute Common Terminology Criteria for AEs [NCI-CTCAE] grade), physical examinations, vital signs, concomitant medication use, and screening for TB. The AEs of interest included malignancies, active TB, opportunistic infections, major adverse $\mathrm{CV}$ events (MACE, defined as CV death, nonfatal myocardial infarction, or nonfatal stroke), clinical laboratory abnormalities, and injection-site reactions. Serum samples were collected at regular intervals through Week 52 of both studies and were analyzed for the presence of antibodies to guselkumab using a validated immunoassay method.

Statistical methods. The descriptive summaries of posthoc safety data reported pooled data across DISCOVER-1 and DISCOVER-2. All patients who received at least 1 dose of study medication were included in the safety assessments, with AEs summarized by actual treatment received. As exposure time to guselkumab varied in different treatment groups as a result of the placebo crossover study design, the number of patients with AEs are reported on the basis of 100 patient-years (PYs) of follow-up. The numbers of AEs through 1 year were also standardized per 100 PYs.

\section{RESULTS}

Patients. A total of 1123 patients were enrolled in DISCOVER-1 and DISCOVER-2; of these, 3 patients discontinued before receiving any study treatment. Thus, the pooled population of patients from the 2 studies included 1120 treated patients: 381 


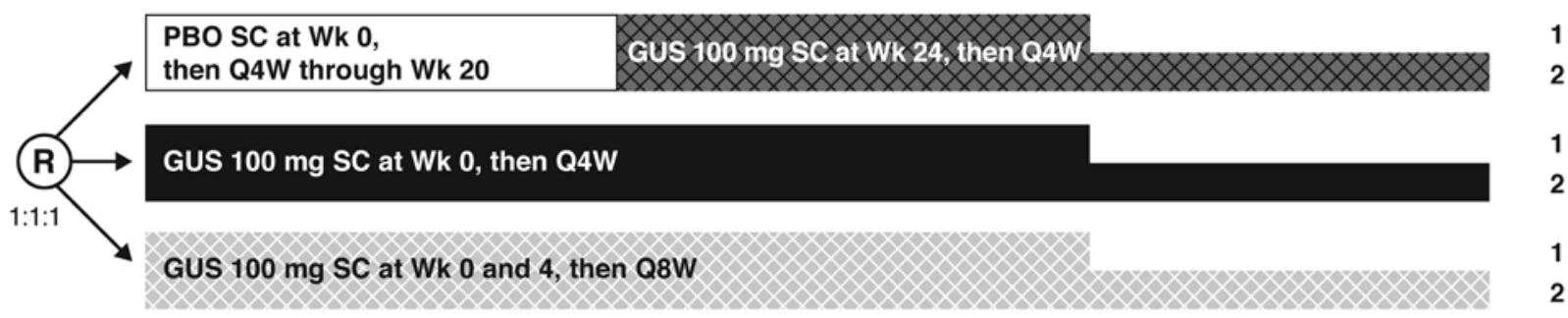

1

1

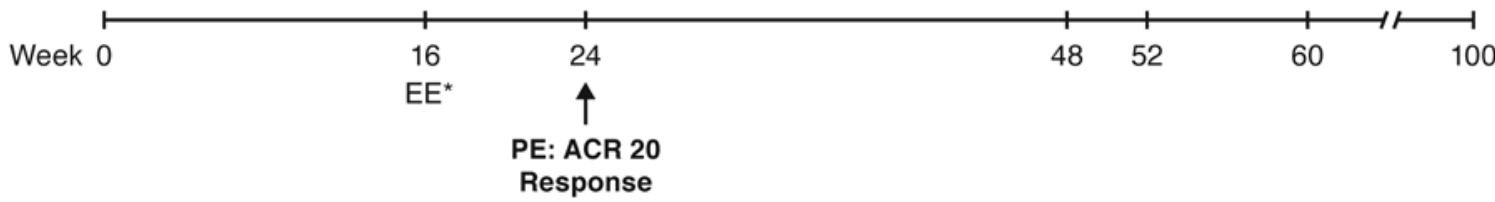

Figure 1. DISCOVER 1 and DISCOVER 2 Study Designs. ${ }^{*}$ Patients were eligible to initiate/increase background medications if they had $<5 \%$ improvement from baseline in both tender and swollen joint counts at Week 16. ACR20: American College of Rheumatology criteria 20\% improvement; EE: early escape; GUS: guselkumab; PBO: placebo; PE: primary endpoint; Q4W: every 4 weeks; Q8W: every 8 weeks; R: randomization; SC: subcutaneous; Wk: week.

from DISCOVER-1 ${ }^{9}$ and 739 from DISCOVER-2. ${ }^{10}$ Patients had an approximate mean age of 47 years and mean PsA duration of 6 years at study outset. Consistent with each study's entrance criteria, patients entered the trials with active PsA (Table 1). Baseline characteristics were generally similar between the studies ${ }^{9,10}$ with the exception of patients in DISCOVER-1 having a longer disease duration, and patients in the DISCOVER-2 trial having higher CRP and numerically higher numbers of swollen and tender joints and greater extent or severity of skin disease assessed using the Psoriasis Area Severity Index.

Detailed patient disposition through 1 year has been reported. ${ }^{9,10,13,14}$ Through Week 24, 3.8\% (14/373) of patients in the guselkumab Q4W group, 3.2\% (12/375) in the guselkumab Q8W group, and 5.4\% (20/372) in the placebo group

Table 1. Pooled baseline characteristics from the DISCOVER-1 and DISCOVER-2 trials.

\begin{tabular}{|c|c|c|c|c|}
\hline & Placebo & $\begin{array}{c}\text { GUS } 100 \mathrm{mg} \\
\text { Q4W }\end{array}$ & $\begin{array}{c}\text { GUS } 100 \mathrm{mg} \\
\text { Q8W }\end{array}$ & $\begin{array}{c}\text { GUS } \\
\text { Combined }^{a}\end{array}$ \\
\hline Patients, $\mathrm{n}$ & 372 & 373 & 375 & 748 \\
\hline Age, yrs & $47.2(11.5)$ & $46.5(11.5)$ & $46.2(11.9)$ & $46.3(11.7)$ \\
\hline Sex, male, n (\%) & $178(48)$ & $208(56)$ & $197(53)$ & $405(54)$ \\
\hline BMI, $\mathrm{kg} / \mathrm{m}^{2}$ & $29.2(6.1)$ & $29.4(5.8)$ & $29.1(6.3)$ & $29.2(6.0)$ \\
\hline PsA disease duration, yrs & $6.3(6.4)$ & $5.9(6.1)$ & $5.6(5.7)$ & $5.7(5.9)$ \\
\hline Swollen joint count $(0-66)$ & $11.5(7.0)$ & $11.4(7.5)$ & $11.4(7.7)$ & $11.4(7.6)$ \\
\hline Tender joint count $(0-68)$ & $21.0(13.5)$ & $20.8(13.6)$ & $19.9(12.8)$ & $20.4(13.2)$ \\
\hline CRP, mg/dL, median (IQR) & $0.9(0.5-2.4)$ & $0.9(0.5-1.9)$ & $1.0(0.5-2.3)$ & $0.9(0.5-2.2)$ \\
\hline HAQ-DI & $1.3(0.6)$ & $1.2(0.6)$ & $1.3(0.6)$ & $1.2(0.6)$ \\
\hline BSA affected by $\mathrm{PsO}, \%$ & $15.4(18.9)$ & $17.1(19.7)$ & $15.7(20.0)$ & $16.4(19.9)$ \\
\hline PASI (0-72) & $8.8(9.5)$ & $10.4(11.2)$ & $9.2(11.1)$ & $9.8(11.1)$ \\
\hline \multicolumn{5}{|l|}{ IGA, VAS $(0-10 \mathrm{~cm})$} \\
\hline$\geq 2, \mathrm{n}(\%)$ & $301(81)$ & $311(83)$ & $295(79)$ & $606(81)$ \\
\hline Previous anti-TNF use ${ }^{\mathrm{b}}, \mathrm{n}(\%)$ & $39(10)$ & $38(10)$ & $41(11)$ & $79(11)$ \\
\hline \multicolumn{5}{|l|}{ Medication use at baseline, $\mathrm{n}(\%)$} \\
\hline MTX & $227(61)$ & $218(58)$ & $209(56)$ & $427(57)$ \\
\hline Oral corticosteroids & $69(19)$ & $62(17)$ & $68(18)$ & $130(17)$ \\
\hline NSAIDs & $245(66)$ & $240(64)$ & $236(63)$ & $476(64)$ \\
\hline
\end{tabular}

Results presented are mean (SD), unless otherwise indicated. ${ }^{a}$ Combined GUS Q4W and Q8W treatment groups. ${ }^{b}$ All patients with previous anti-TNF use were in the DISCOVER-1 trial. BSA: body surface area; CRP: C-reactive protein; GUS: guselkumab; HAQ-DI: Health Assessment Questionnaire-Disability Index; IGA: investigator global assessment of psoriasis (cleared $=0$, minimal $=1$, mild $=2$, moderate $=3$, severe $=4$ ); MTX: methotrexate; NSAID: nonsteroidal antiinflammatory drug; PASI: Psoriasis Area And Severity Index; PsA: psoriatic arthritis; PsO: psoriasis; Q4W: every 4 weeks; Q8W: every 8 weeks; TNF: tumor necrosis factor; VAS: visual analog scale. 
discontinued the study agent. AEs leading to study agent discontinuation occurred in $1.9 \%, 1.3 \%$, and $1.6 \%$ of patients, respectively, in the guselkumab $\mathrm{Q} 4 \mathrm{~W}$, guselkumab $\mathrm{Q} 8 \mathrm{~W}$, and placebo groups.

Among patients continuing treatment at Week 24, 2.2\% $(8 / 359)$ of patients in the guselkumab Q4W group, 3.7\% $(13 / 363)$ in the guselkumab Q8W group, and 4.8\% (17/352) of patients who crossed over from placebo to guselkumab Q4W at Week 24 discontinued the study agent through 1 year. AEs leading to study agent discontinuation occurred in $0.3 \%, 0.6 \%$, and $1.7 \%$ patients, respectively, in the guselkumab $\mathrm{Q} 4 \mathrm{~W}$, guselkumab Q8W, and placebo to guselkumab crossover groups.

AEs during the placebo-controlled period through Week 24. Through Week 24, the numbers of patients with $\geq 1 \mathrm{AE}$ per 100 PYs (95\% CI) were 153.7 (132.3-177.7) for guselkumab 100 mg Q4W, 147.7 (127.0-170.7) for guselkumab $100 \mathrm{mg}$ Q8W, and 142.8 (122.5-165.6) for placebo. The numbers of patients experiencing serious AEs (SAEs; 4.4 and 7.1/100 PYs), AEs leading to discontinuation of study agent (3.8 and 4.1/100 PYs), infections (49.5 and 49.9/100 PYs), and serious infections (1.2 and 1.7/100 PYs) were similar in patients treated with the combined guselkumab and placebo regimens, respectively (Table 2). Results were consistent when assessing the numbers of events/100 PYs (Supplementary
Table 1, available with the online version of this article). The most common infections were nasopharyngitis (combined guselkumab Q4W and Q8W groups, $\mathrm{n}=45$ [6.0\%]; placebo, $\mathrm{n}=17[4.6 \%])$, upper respiratory tract infection $(\mathrm{n}=38$ [5.1\%]; placebo, $\mathrm{n}=17$ [4.6\%]), and bronchitis $(\mathrm{n}=17$ [2.3\%]; placebo, $\mathrm{n}=4[1.1 \%])$.

AEs reported with guselkumab through 1 year. At 1 year, time-adjusted incidences of AEs and SAEs remained stable in both guselkumab treatment groups. Among patients treated with placebo who crossed over to guselkumab $100 \mathrm{mg}$ Q4W at Week 24, time-adjusted incidences of AEs were generally comparable to those of patients originally randomized to either dose regimen of guselkumab and treated for 1 year (Table 2). Infections were the most common class of AEs through 1 year, with nasopharyngitis, upper respiratory infections, and bronchitis occurring in $8.4 \%, 7.1 \%$, and $3.4 \%$ of the 1100 patients treated with guselkumab, respectively, including those who crossed over from placebo at Week 24. As through Week 24, results through 1 year were consistent when assessing the numbers of events/100 PYs (Supplementary Table 1, available with the online version of this article).

AEs of interest. Two patients treated with placebo died through Week 24 (cardiac failure, pneumonia) ${ }^{9,10}$; no patients treated with guselkumab died through 1 year. ${ }^{13,14}$ Serious infections were

Table 2. Number of patients with AEs per 100 PYs.

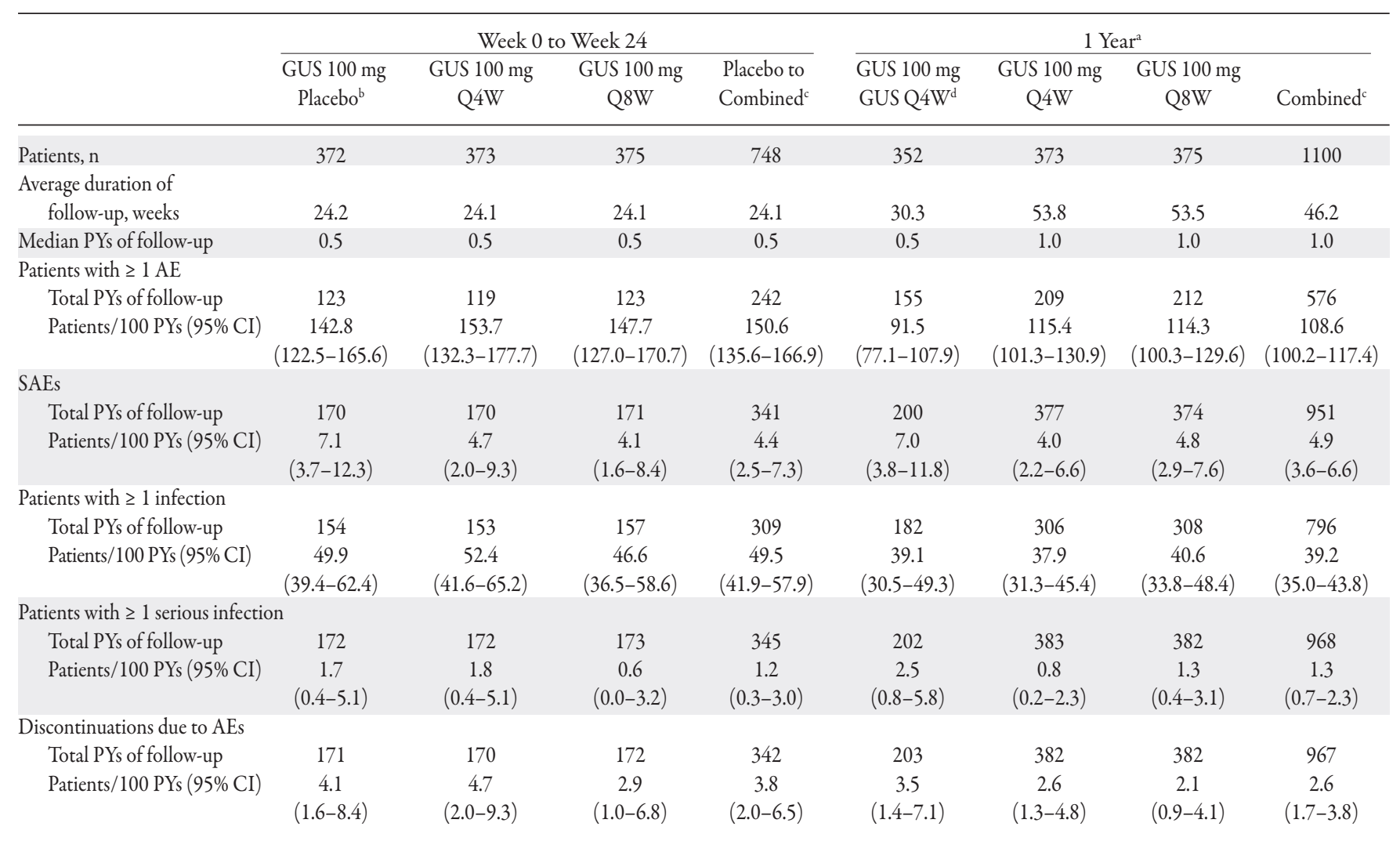

a Through Week 60 for DISCOVER-1 and Week 52 for DISCOVER-2. ${ }^{\text {b }}$ For patients in the PBO group who crossed over to GUS Q4W, only data prior to first administration of GUS are included in this group. ${ }^{c}$ Combined patients treated with GUS Q4W and Q8W (including patients crossed over from PBO for 1-year results). ${ }^{\mathrm{d}}$ For patients in the PBO group who crossed over to GUS Q4W, only data on and after first administration of GUS were included in this group. AE: adverse event; GUS: guselkumab; PBO: placebo; PY: patient-year; Q4W: every 4 weeks; Q8W: every 8 weeks; SAE: serious adverse event. 
uncommon across treatment groups (Table 2), with no cases of active TB or opportunistic infections reported through 1 year. One case of nonserious oral thrush was reported in a patient treated with guselkumab with a history of asthma and concomitant inhaled corticosteroid use. ${ }^{14}$ One case each of IBD and iridocyclitis occurred in a patient treated with placebo.

Malignancies occurred in 4 patients and have been previously reported in detail. One patient receiving placebo was diagnosed with renal cell carcinoma, ${ }^{10}$ and another patient who crossed over from placebo to guselkumab $100 \mathrm{mg}$ Q $4 \mathrm{~W}$ at Week 24 had squamous cell skin carcinoma and malignant melanoma (both reported at Week 36). ${ }^{14}$ Among patients receiving guselkumab $100 \mathrm{mg}$ Q8W, one was diagnosed with multiple myeloma 15 days after the first guselkumab injection, ${ }^{9}$ and another (with a preexisting skin lesion of pigmented macule) was diagnosed with melanoma in situ. ${ }^{13}$ No increase in malignancy was observed from Week 24 to 1 year (Table 3 ). The MACE events that occurred in 2 patients prior to Week 24 (i.e., the aforementioned event of cardiac failure in a patient receiving placebo 9 and ischemic stroke in 1 patient receiving guselkumab $100 \mathrm{mg} \mathrm{Q}_{4} \mathrm{~W}^{10}$ ) were also previously reported. The latter patient had hypertension, hyperlipidemia, and diabetes at baseline.

The incidence of injection-site reactions through Week 24 was low in both guselkumab treatment groups (1.1\% and $1.3 \%$ in the Q4W and Q8W groups, respectively; Table 3). Through 1 year, the rate of injection-site reactions remained low $(2.4 \%$ and $1.6 \%$ in the Q4W and Q8W groups, respectively). Most injection-site reactions were mild, and the most common reaction was erythema. Two moderate injection-site reactions occurred in patients receiving guselkumab $100 \mathrm{mg}$ Q $4 \mathrm{~W}$, and both led to discontinuation of study treatment. ${ }^{13}$

Laboratory investigations. Results of laboratory investigations within the individual studies have been previously reported through 1 year..$^{9,10,13,14}$ During the placebo-controlled periods of the DISCOVER trials, elevations in serum alanine aminotransferase (ALT) and/or aspartate aminotransferase (AST) appeared to be more common with guselkumab than placebo. Pooled incidences of hepatic aminotransferase elevations through 1 year of treatment with either regimen of guselkumab were generally consistent with those through Week 24, considering the additional duration of follow-up (Table 4). The majority of these elevations were NCI-CTCAE Grade 1. No patients treated with guselkumab experienced Grade 4 ALT or AST elevations through 1 year. Whereas Grade 2 and 3 elevations were more common in the Q4W group compared with the Q8W group, most were transient and did not result in discontinuation (see exceptions in the following section), and none was associated with increases in bilirubin $>2$ times the upper limit of normal. Confounding factors were present in the majority of patients with Grade 2 or 3 elevations in hepatic transaminases, such as an underlying medical condition, obesity, concomitant alcohol use, latent TB treatment, or concomitant treatment with DMARDs or NSAIDs that are associated with liver injury.

Through 1 year, Grade 1 or higher elevated ALT levels were slightly more common in pooled patients with baseline use of methotrexate (MTX; guselkumab Q4W: 38.7\%, guselkumab Q8W: 39.0\%), compared with pooled patients without baseline use of MTX (Q4W: 35.9\%, Q8W: 32.5\%; Table 4). Grade 1 or higher increased AST levels were also slightly more common in patients with baseline use of MTX (Q4W: 30.1\%; Q8W: 27.1\%) compared with patients without use of baseline MTX (Q4W: 25.6\%; Q8W: 25.3\%).

Four patients receiving guselkumab $100 \mathrm{mg}$ Q4W discontinued treatment as a result of hepatobiliary AEs or elevated transaminases. ${ }^{13}$ Three of the patients were also receiving isoniazid; 2 had drug (isoniazid)-induced liver injury, and the third patient had elevated transaminase levels with active alcohol use, with hepatology evaluation revealing chronic pancreatitis, chronic cholecystitis, and fatty liver disease. Isoniazid was discontinued in these patients, and transaminase levels declined in all 3. The patient who was not receiving isoniazid had acute hepatitis B; of note, the patient's family history included hepatitis B-positive status of the spouse.

Table 3. Adverse events of interest.

\begin{tabular}{|c|c|c|c|c|c|c|c|c|}
\hline & Placebo $^{b}$ & $\begin{array}{c}\text { GUS } 100 \mathrm{mg} \\
\text { Q4W }\end{array}$ & $\begin{array}{c}\text { GUS } 100 \mathrm{mg} \\
\text { Q8W }\end{array}$ & $\begin{array}{l}\text { GUS } 100 \mathrm{mg} \\
\text { Combined }^{\mathrm{c}}\end{array}$ & $\begin{array}{l}\text { Placebo to } \\
\text { GUS Q4WW }\end{array}$ & $\begin{array}{c}\text { GUS } 100 \mathrm{mg} \\
\text { Q4W }\end{array}$ & $\begin{array}{c}\text { GUS } 100 \mathrm{mg} \\
\text { Q8W }\end{array}$ & $\begin{array}{l}\text { GUS } 100 \mathrm{mg} \\
\text { Combined }^{\mathrm{c}}\end{array}$ \\
\hline Patients, $\mathrm{n}$ & 372 & 373 & 375 & 748 & 352 & 373 & 375 & 1100 \\
\hline Death & $2(0.5)$ & 0 & 0 & 0 & 0 & 0 & 0 & 0 \\
\hline Malignancy & $1(0.3)$ & 0 & $2(0.5)$ & $2(0.3)$ & $1(0.3)$ & 0 & $2(0.5)$ & $3(0.3)$ \\
\hline TB & 0 & 0 & 0 & 0 & 0 & 0 & 0 & 0 \\
\hline IBD & $1(0.3)$ & 0 & 0 & 0 & 0 & 0 & 0 & 0 \\
\hline Injection-site reaction & $1(0.3)$ & $4(1.1)$ & $5(1.3)$ & $9(1.2)$ & $4(1.1)$ & $9(2.4)$ & $6(1.6)$ & $19(1.7)$ \\
\hline
\end{tabular}

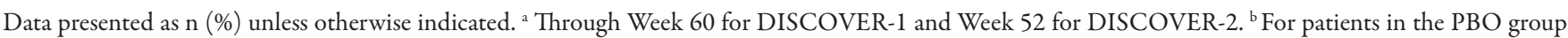
who crossed over to GUS Q4W, only data prior to first administration of GUS are included in this group. ${ }^{\circ}$ Combined patients treated with GUS Q4W and Q8W (including patients who crossed over from PBO for 1-year results). ${ }^{\mathrm{d}}$ For patients in the PBO group who crossed over to GUS Q4W, only data on and after first administration of GUS were included in this group. GUS: guselkumab; IBD: inflammatory bowel disease; MACE: major adverse cardiovascular events (cardiovascular death, nonfatal myocardial infarction, or nonfatal stroke); OI: opportunistic infection; PBO: placebo; Q4W: every 4 weeks; Q8W: every 8 weeks; TB: tuberculosis. 
Table 4. Proportions of patients with clinical laboratory abnormalities according to NCI-CTCAE grade.

\begin{tabular}{|c|c|c|c|c|c|c|c|c|c|}
\hline & \multicolumn{4}{|c|}{ Week 0 to Week 24} & \multicolumn{5}{|c|}{1 Year $^{\mathrm{a}}$} \\
\hline & $\mathrm{PBO}^{\mathrm{b}}$ & $\begin{array}{l}\text { GUS } 100 \mathrm{mg} \\
\text { Q4W }\end{array}$ & $\begin{array}{l}\text { GUS } 100 \mathrm{mg} \\
\text { Q8W }\end{array}$ & $\begin{array}{l}\text { GUS } 100 \mathrm{mg} \\
\text { Combined }^{\mathrm{c}}\end{array}$ & $\begin{array}{c}\text { PBO to } \\
\text { GUS Q4W }\end{array}$ & $\begin{array}{l}\text { GUS } 100 \mathrm{mg} \\
\text { Q4W }\end{array}$ & $\begin{array}{c}\text { GUS } 100 \mathrm{mg} \\
\text { Q8W }\end{array}$ & $\begin{array}{c}\text { GUS } 100 \mathrm{mg} \\
\text { Q4W Combined }\end{array}$ & $\begin{array}{l}\text { GUS } 100 \mathrm{mg} \\
\text { Combined }^{c}\end{array}$ \\
\hline $\mathrm{n}$ & 370 & 371 & 373 & 744 & 351 & 371 & 373 & 722 & 1095 \\
\hline Grade 1 & $111(30.0)$ & $130(35.0)$ & $105(28.2)$ & $235(31.6)$ & $90(25.6)$ & $153(41.2)$ & $125(33.5)$ & $243(33.7)$ & $368(33.6)$ \\
\hline Grade 4 & $1(0.3)$ & 0 & 0 & 0 & 0 & 0 & 0 & 0 & 0 \\
\hline \multicolumn{10}{|c|}{ ALT increased, baseline MTX } \\
\hline $\mathrm{n}$ & 225 & 216 & 207 & 423 & 213 & 216 & 207 & 429 & 636 \\
\hline Grade 1 & $78(34.7)$ & $82(38.0)$ & $66(31.9)$ & $148(35.0)$ & $57(26.8)$ & $92(42.6)$ & $74(35.7)$ & $149(34.7)$ & $223(35.1)$ \\
\hline Grade 2 & $5(2.2)$ & $7(3.2)$ & $3(1.4)$ & $10(2.4)$ & $5(2.3)$ & $10(4.6)$ & $4(1.9)$ & $15(3.5)$ & $19(3.0)$ \\
\hline Grade 3 & $1(0.4)$ & $2(0.9)$ & $2(1.0)$ & $4(0.9)$ & 0 & $2(0.9)$ & $3(1.4)$ & $2(0.5)$ & $5(0.8)$ \\
\hline Grade 2 & 0 & $3(1.9)$ & $1(0.6)$ & $4(1.2)$ & $2(1.4)$ & $7(4.5)$ & $2(1.2)$ & $9(3.1)$ & $11(2.4)$ \\
\hline Grade 3 & $1(0.7)$ & $2(1.3)$ & $1(0.6)$ & $3(0.9)$ & 0 & $2(1.3)$ & $1(0.6)$ & $2(0.7)$ & $3(0.7)$ \\
\hline Grade 4 & $1(0.7)$ & 0 & 0 & 0 & 0 & 0 & 0 & 0 & 0 \\
\hline \multicolumn{10}{|c|}{ AST increased ${ }^{f}$} \\
\hline $\mathrm{n}$ & 370 & 371 & 373 & 744 & 351 & 371 & 373 & 722 & 1095 \\
\hline Grade 1 & $74(20.0)$ & $80(21.6)$ & $70(18.8)$ & $150(20.2)$ & $74(21.1)$ & $103(27.8)$ & $85(22.8)$ & $177(24.5)$ & $262(23.9)$ \\
\hline Grade 2 & $2(0.5)$ & $6(1.6)$ & $6(1.6)$ & $12(1.6)$ & $6(1.7)$ & $14(3.8)$ & $11(2.9)$ & $20(2.8)$ & $31(2.8)$ \\
\hline Grade 3 & $4(1.1)$ & $6(1.6)$ & $2(0.5)$ & $8(1.1)$ & $1(0.3)$ & $6(1.6)$ & $2(0.5)$ & $7(1.0)$ & $9(0.8)$ \\
\hline Grade 4 & 0 & 0 & 0 & 0 & 0 & 0 & 0 & 0 & 0 \\
\hline \multicolumn{10}{|c|}{ AST increased, baseline $\mathrm{MTX}^{\mathrm{f}}$} \\
\hline $\mathrm{n}$ & 225 & 216 & 207 & 423 & 213 & 216 & 207 & 429 & 636 \\
\hline Grade 1 & $54(24.0)$ & $56(25.9)$ & $41(19.8)$ & $97(22.9)$ & $46(21.6)$ & $68(31.5)$ & $50(24.2)$ & $114(26.6)$ & $164(25.8)$ \\
\hline \multicolumn{10}{|c|}{ AST increased, no baseline MTX ${ }^{f}$} \\
\hline $\mathrm{n}$ & 145 & 155 & 166 & 321 & 138 & 155 & 166 & 293 & 459 \\
\hline Grade 1 & $20(13.8)$ & $24(15.5)$ & $29(17.5)$ & $53(16.5)$ & $28(20.3)$ & $35(22.6)$ & $35(21.1)$ & $63(21.5)$ & $98(21.4)$ \\
\hline Grade 2 & $1(0.7)$ & $2(1.3)$ & $3(1.8)$ & $5(1.6)$ & $2(1.4)$ & $5(3.2)$ & $6(3.6)$ & $7(2.4)$ & $13(2.8)$ \\
\hline Grade 3 & $3(2.1)$ & $4(2.6)$ & $1(0.6)$ & $5(1.6)$ & $1(0.7)$ & $4(2.6)$ & $1(0.6)$ & $5(1.7)$ & $6(1.3)$ \\
\hline Grade 4 & 0 & 0 & 0 & 0 & 0 & 0 & 0 & 0 & 0 \\
\hline \multicolumn{10}{|c|}{ Neutrophil count decreased ${ }^{f}$} \\
\hline $\mathrm{n}$ & 370 & 371 & 373 & 744 & 351 & 371 & 373 & 722 & 1095 \\
\hline Grade 1 & $12(3.2)$ & $22(5.9)$ & $21(5.6)$ & $43(5.8)$ & $15(4.3)$ & $29(7.8)$ & $36(9.7)$ & $44(6.1)$ & $80(7.3)$ \\
\hline Grade 2 & $3(0.8)$ & $6(1.6)$ & $6(1.6)$ & $12(1.6)$ & $3(0.9)$ & $12(3.2)$ & $10(2.7)$ & $15(2.1)$ & $25(2.3)$ \\
\hline Grade 3 & $1(0.3)$ & 0 & 0 & 0 & $2(0.6)$ & $1(0.3)$ & $2(0.5)$ & $3(0.4)$ & $5(0.5)$ \\
\hline Grade 4 & 0 & $1(0.3)$ & 0 & $1(0.1)$ & 0 & $1(0.3)$ & 0 & $1(0.1)$ & $1(0.1)$ \\
\hline
\end{tabular}

Data presented as n (\%) unless otherwise indicated. ${ }^{\text {a }}$ Through week 60 for DISCOVER-1 and week 52 for DISCOVER-2. ${ }^{\mathrm{b}}$ For patients in the PBO group who crossed over to GUS Q4W, only data prior to the first administration of GUS are included in this group. ${ }^{c}$ Combined patients treated with GUS Q4W and Q8W (including patients who crossed over from PBO for 1-year results). ${ }^{\mathrm{d}}$ For patients in the PBO group who crossed over to GUS Q4W, only data on and after first administration of GUS were included in this group. ${ }^{\mathrm{e}}$ Combined patients treated with GUS Q4W (including patients who crossed over from PBO). ${ }^{\mathrm{f}}$ NCI-CTCAE grades. ALT: alanine aminotransferase; AST: aspartate aminotransferase; GUS: guselkumab; MTX: methotrexate; NCI-CTCAE: National Cancer Institute Common Terminology Criteria for Adverse Events; PBO: placebo; Q4W: every 4 weeks; Q8W: every 8 weeks.

Decreased neutrophil counts were slightly more common in patients treated with guselkumab than those treated with placebo, with no increase seen from Week 24 to 1 year (Table 4). Most cases were NCI-CTCAE Grade 1; those Grade 2 or greater were reversible and did not result in treatment discontinuation. The decreased neutrophil counts were not associated with infection, except for 1 patient who experienced mild nasopharyngitis that lasted 5 days after a decreased neutrophil count of Grade 2 was observed.

Immunogenicity. Antibodies to guselkumab were detected in the serum of $4.5 \%(49 / 1094)$ of pooled patients treated with guselkumab with appropriate samples through Week 52 of the 
Table 5. Proportions of GUS-treated patients with antibodies to GUS.

\begin{tabular}{|c|c|c|c|c|c|c|c|}
\hline & \multicolumn{3}{|c|}{ Through Week 24} & \multicolumn{4}{|c|}{ Through Week 52} \\
\hline & $\begin{array}{c}\text { GUS } 100 \mathrm{mg} \\
\text { Q4W }\end{array}$ & $\begin{array}{c}\text { GUS } 100 \mathrm{mg} \\
\text { Q8W }\end{array}$ & $\begin{array}{l}\text { GUS } 100 \mathrm{mg} \\
\text { Combined }^{a}\end{array}$ & $\begin{array}{c}\text { PBO to } \\
\text { GUS Q4W }\end{array}$ & $\begin{array}{c}\text { GUS } 100 \mathrm{mg} \\
\text { Q4W }\end{array}$ & $\begin{array}{c}\text { GUS } 100 \mathrm{mg} \\
\text { Q8W }\end{array}$ & $\begin{array}{r}\text { GUS } 100 \mathrm{mg} \\
\text { Combined }^{\mathrm{a}}\end{array}$ \\
\hline
\end{tabular}

${ }^{a}$ Included all patients who received at least 1 dose of GUS. ${ }^{\mathrm{b}}$ Included patients in the PBO group who crossed over to GUS Q4W at Week $24 .{ }^{\mathrm{c}}$ Presence of antibodies to GUS in serum samples of GUS-treated patients was assessed using a validated immunoassay method. Denominator is patients with appropriate samples. GUS: guselkumab; PBO: placebo; Q4W: every 4 weeks; Q8W: every 8 weeks.

DISCOVER trials, with similar incidence between guselkumab dosing regimens (Table 5). No association was noted between the development of antibodies to guselkumab and the occurrence of injection-site reactions, albeit the number of positive patients was small. Through Week 52, 10.2\% (5/49) of patients with aantibodies to guselkumab $(0.5 \%$ of all patients treated with guselkumab) had neutralizing antibodies (data not shown).

\section{DISCUSSION}

Herein, we report the pooled safety results through 1 year in 1120 patients from DISCOVER-1 and DISCOVER-2, the placebo-controlled, phase III studies of SC guselkumab $100 \mathrm{mg}$ Q4W or Q8W conducted in patients with active PsA. Findings were consistent with those previously reported for each trial through Week $24^{9,10}$ and through 1 year, ${ }^{13,14}$ as well as with the long-term safety results through 4 years of guselkumab treatment in the VOYAGE-1 and VOYAGE-2 trials conducted in patients with moderate-to-severe $\mathrm{PsO}_{\mathrm{S}}{ }^{19,20}$ Through Week 24, time-adjusted rates (per $100 \mathrm{PYs}$ ) of AEs, SAEs, infections, serious infections, and discontinuations as the result of an AE were similar across the placebo and guselkumab treatment groups. Through 1 year, the rates for these $\mathrm{AE}$ categories remained stable. In addition, no patients treated with guselkumab developed uveitis, active TB, an opportunistic infection (noting 1 case of nonserious oral thrush), or IBD through 1 year. As well, incidences of malignancy and MACE were similar across treatment groups through Week 24, with no increase through 1 year. Two deaths occurred through 1 year, both in patients receiving placebo. Injection-site reactions were uncommon, as was the development of neutralizing antibodies to guselkumab. Elevated serum hepatic transaminases and decreased neutrophil counts were generally mild and transient through 1 year.

Biologics have provided a highly effective alternative for treating the signs and symptoms of PsA and $\mathrm{PsO}$, including anti-TNF agents and monoclonal antibodies targeting IL-12/23, IL-17, and IL-23. The safety profiles of anti-TNF agents and ustekinumab, a monoclonal antibody targeting IL-12/23, are well established, ${ }^{16,21}$ with IL-17 antibodies accruing longer-term data more recently. While generally not associated with chronic organ damage, biologics can have significant AEs. ${ }^{17,22}$

Concerns associated with anti-TNF therapies include an increased risk of serious infections, particularly TB and opportunistic infections, new-onset or worsening of heart failure, hypersensitivity reactions, and malignancy. ${ }^{23}$ Prescribing information for the IL-12/23 inhibitor ustekinumab includes warnings and precautions for infections, malignancy, and hypersensitivity reactions..$^{24}$ However, long-term registry data in $\mathrm{PsO}$ suggest the risk of serious infection with ustekinumab may be lower than with anti-TNF agents. ${ }^{25}$ IL-17 antibodies are associated with a risk of infection, hypersensitivity, and new-onset or exacerbation of IBD. ${ }^{26,27}$ Agents targeting IL-23p19 represent the newest class of biologics approved for PsA. The prescribing information for guselkumab cautions on infection and hypersensitivity. ${ }^{1}$ Prescribing information for ustekinumab and anti-IL-17 and -IL-23 agents approved for PsA all recommend TB testing and prophylaxis. ${ }^{1,24,26,27}$

Longer-term clinical data and registries have further clarified the actual risk of these $\mathrm{AEs}{ }^{25,28}$ For example, the increased risk of TB (new-onset TB and reactivation of latent TB infection [LTBI]) with anti-TNF agents is well established and derives from the pivotal role of TNF in maintaining granuloma integrity. ${ }^{29}$ TB screening and treatment of LTBI are recommended before initiating therapy with any biologic approved for PsA, implying an increased risk associated with treatment. However, associations with active TB and LTBI are generally less common with other biologic classes for immune-mediated diseases than with anti-TNF agents. ${ }^{30,31,32,33}$ No cases of new-onset TB or reactivation of LTBI occurred through 1 year of the DISCOVER-1 and DISCOVER-2 trials. This is consistent with the pivotal phase III PsO trials of guselkumab that included an active comparator arm, adalimumab. ${ }^{8,11}$ In the $\mathrm{PsO}$ trials, among patients who had LTBI and received prophylactic treatment, no cases of active TB were reported in any treatment group. Among patients with no TB at baseline, no new cases of active TB developed in patients treated with either guselkumab or placebo, whereas 2 patients with $\mathrm{PsO}$ treated with adalimumab developed active TB. ${ }^{34}$ While further long-term safety data are awaited, choice of biologic treatment for PsA, particularly in TB endemic regions, should include consideration of the current data on $\mathrm{TB}$ reactivation.

In the phase III studies of anti-IL-17 antibodies for PsA, increased rates of mucocutaneous Candida infections, rarely serious, occurred in the active treatment arms, ${ }^{35,36,37}$ continued during longer treatment periods, ${ }^{38,39}$ and are consistent with the $\mathrm{PsO}$ phase III studies. ${ }^{40,41,42,43}$ The increased rate is likely attributable to the role of IL-17 in host defense against fungal infections, particularly at mucosal sites. ${ }^{44}$ In contrast, in DISCOVER-1 and DISCOVER-2, no opportunistic infections occurred through 1 year (noting 1 case of nonserious oral thrush). New-onset or exacerbation of IBD has also been reported in clinical trials 
of anti-IL-17 antibodies. Clinical trials for IL-17 blockade to treat Crohn disease were either unsuccessful or stopped early as a result of exacerbation of disease. ${ }^{45,46}$ In clinical trials of a monoclonal antibody against IL-17A for PsO or PsA, cases of new-onset or exacerbation of IBD have been reported. ${ }^{38,47,48}$ In the ECLIPSE study, which compared guselkumab with secukinumab in patients with $\mathrm{PsO}, 3$ (1\%) patients in the secukinumab group compared with none in the guselkumab group reported an event of Crohn disease through Week $56 .{ }^{18}$ In the current pooled analysis of the phase III guselkumab PsA studies of 1120 patients through 1 year, no cases of IBD were reported among patients treated with guselkumab.

Elevations in hepatic aminotransferases appeared to be more common among patients treated with guselkumab (higher with Q4W than Q8W dosing) than patients treated with placebo. The elevations were generally of low toxicity grade, transient, and not associated with clinically significant increases in bilirubin. Elevations of Grade 2 or 3 were mostly associated with confounding factors such as prior and/or concomitant use of medications associated with liver injury. In general, increases in ALT and AST were more common in patients with baseline MTX use. Decreased neutrophil counts were also somewhat more common with guselkumab compared with placebo, although most were of low toxicity grade, transient, and not associated with infection.

Injection-site reactions were uncommon through 1 year, occurring in fewer than $2 \%$ of patients treated with guselkumab. While nearly all were mild, the 2 moderate injection-site reactions led to discontinuation of guselkumab. The overall incidence of antibodies to guselkumab remained low (4.5\%) through Week 52, with no apparent association between their development and the occurrence of injection-site reactions. Of the antibodies detected, $10 \%$ were neutralizing antibodies. However, because the number of patients who were positive for antibodies to guselkumab was small, no definitive conclusions about the influence of antibodies on pharmacokinetics or pharmacodynamics of guselkumab can be drawn.

This pooled analysis is limited by a 1 -year follow-up time. However, the upcoming 2-year results from DISCOVER-2 will provide longer-term safety results. In addition, the studies were not powered to detect rare events.

In conclusion, the results of this pooled safety analysis of the DISCOVER-1 and DISCOVER-2 trials indicate that guselkumab $100 \mathrm{mg}$, given either Q4W or Q8W, was generally well tolerated in this population of patients with active PsA. Further, the guselkumab safety profile in patients with PsA through 1 year is consistent with that in patients with $\mathrm{PsO}$ who received up to 5 years of guselkumab. ${ }^{49}$

\section{ACKNOWLEDGMENT}

The authors thank Elizabeth Rosenberg, PhD, and Cynthia Guzzo, MD, of Kelly Services, and Rebecca Clemente, PhD, of Janssen Scientific Affairs for writing support in the preparation of this manuscript.

\section{ONLINE SUPPLEMENT}

Supplementary material accompanies the online version of this article.

\section{REFERENCES}

1. Tremfya [package insert]. Horsham, PA: Janssen Biotech, Inc.; 2020.

2. Al-Salama ZT, Scott LJ. Guselkumab: a review in moderate to severe plaque psoriasis. Am J Clin Dermatol 2018;19:907-18.

3. Abdo AIK, Tye GJ. Interleukin 23 and autoimmune diseases: current and possible future therapies. Inflamm Res 2020;69:463-80.

4. Bravo A, Kavanaugh A. Bedside to bench: defining the immunopathogenesis of psoriatic arthritis. Nat Rev Rheumatol 2019;15:645-56.

5. Girolomoni G, Strohal R, Puig L, Bachelez H, Barker J, Boehncke WH, et al. The role of IL-23 and the IL-23/TH 17 immune axis in the pathogenesis and treatment of psoriasis. J Eur Acad Dermatol Venereol 2017;31:1616-26.

6. Aggarwal S, Ghilardi N, Xie MH, de Sauvage FJ, Gurney AL. Interleukin-23 promotes a distinct $\mathrm{CD} 4 \mathrm{~T}$ cell activation state characterized by the production of interleukin-17. J Biol Chem 2003;278:1910-4.

7. Wilson NJ, Boniface K, Chan JR, McKenzie BS, Blumenschein WM, Mattson JD, et al. Development, cytokine profile and function of human interleukin 17-producing helper T cells. Nat Immunol 2007;8:950-7.

8. Blauvelt A, Papp KA, Griffiths CEM, Randazzo B, Wasfi Y, Shen YK, et al. Efficacy and safety of guselkumab, an anti-interleukin-23 monoclonal antibody, compared with adalimumab for the continuous treatment of patients with moderate to severe psoriasis: results from the phase III, double-blinded, placebo- and active comparator-controlled VOYAGE 1 trial. J Am Acad Dermatol 2017;76:405-17.

9. Deodhar A, Helliwell PS, Boehncke WH, Kollmeier AP, Hsia EC, Subramanian RA, et al. Guselkumab in patients with active psoriatic arthritis who were biologic-naive or had previously received TNF $\alpha$ inhibitor treatment (DISCOVER-1): a double-blind, randomised, placebo-controlled phase 3 trial. Lancet 2020;395:1115-25.

10. Mease PJ, Rahman P, Gottlieb AB, Kollmeier AP, Hsia EC, Xu XL, et al. Guselkumab in biologic-naive patients with active psoriatic arthritis (DISCOVER-2): a double-blind, randomised, placebo-controlled phase 3 trial. Lancet 2020;395:1126-36.

11. Reich K, Armstrong AW, Foley P, Song M, Wasfi Y, Randazzo B, et al. Efficacy and safety of guselkumab, an anti-interleukin-23 monoclonal antibody, compared with adalimumab for the treatment of patients with moderate to severe psoriasis with randomized withdrawal and retreatment: results from the phase III, double-blind, placebo- and active comparator-controlled VOYAGE 2 trial. J Am Acad Dermatol 2017;76:418-31.

12. Ritchlin CT, Colbert RA, Gladman DD. Psoriatic arthritis. N Engl J Med 2017;376:957-70.

13. McInnes IB, Rahman P, Gottlieb AB, Hsia EC, Kollmeier AP, Chakravarty SD, et al. Efficacy and safety of guselkumab, an interleukin-23p19-specific monoclonal antibody, through one year in biologic-naive patients with psoriatic arthritis. Arthritis Rheumatol 2021;73:604-16.

14. Ritchlin CT, Helliwell PS, Boehncke WH, Soriano ER, Hsia EC, Kollmeier AP, et al. Guselkumab, an inhibitor of the IL-23p19 subunit, provides sustained improvement in signs and symptoms of active psoriatic arthritis: 1 year results of a phase III randomised study of patients who were biologic-naive or TNF $\alpha$ inhibitor-experienced. RMD Open 2021;7;e001457.

15. Costello R, David T, Jani M. Impact of adverse events associated with medications in the treatment and prevention of rheumatoid arthritis. Clin Ther 2019;41:1376-96.

16. Girolomoni G, Altomare G, Ayala F, Berardesca E, Calzavara-Pinton P, Chimenti S, et al. Safety of anti-TNF $\alpha$ agents in the treatment of psoriasis and psoriatic arthritis. Immunopharmacol Immunotoxicol 2012;34:548-60. 
17. Kamata M, Tada Y. Efficacy and safety of biologics for psoriasis and psoriatic arthritis and their impact on comorbidities: a literature review. Int J Mol Sci 2020;21:1690.

18. Reich K, Armstrong AW, Langley RG, Flavin S, Randazzo B, Li S, et al. Guselkumab versus secukinumab for the treatment of moderate-to-severe psoriasis (ECLIPSE): results from a phase 3, randomised controlled trial. Lancet 2019;394:831-9.

19. Griffiths CEM, Papp KA, Song M, Miller M, You Y, Shen YK, et al. Continuous treatment with guselkumab maintains clinical responses through 4 years in patients with moderate-to-severe psoriasis: results from VOYAGE 1. J Dermatolog Treat 2020;13:1-9.

20. Reich K, Griffiths CEM, Gordon KB, Papp KA, Song M, Randazzo $\mathrm{B}$, et al. Maintenance of clinical response and consistent safety profile with up to 3 years of continuous treatment with guselkumab: results from the VOYAGE 1 and VOYAGE 2 trials. J Am Acad Dermatol 2020;82:936-45.

21. Ghosh S, Gensler LS, Yang Z, Gasink C, Chakravarty SD, Farahi K, et al. Ustekinumab safety in psoriasis, psoriatic arthritis, and Crohn's disease: an integrated analysis of phase II/III clinical development programs. Drug Saf 2019;42:751-68.

22. Armstrong AW, Read C. Pathophysiology, clinical presentation, and treatment of psoriasis: a review. JAMA 2020;323:1945-60.

23. Atzeni F, Nucera V, Gerratana E, Cirillo M, Marino F, Miceli G, et al. Concerns about the safety of anti-TNF agents when treating rheumatic diseases. Expert Opin Drug Saf 2020;19:695-705.

24. Stelara [package insert]. Horsham, PA: Janssen Biotech, Inc.; 2019.

25. Papp K, Gottlieb AB, Naldi L, Pariser D, Ho V, Goyal K, et al. Safety surveillance for ustekinumab and other psoriasis treatments from the Psoriasis Longitudinal Assessment and Registry (PSOLAR). J Drugs Dermatol 2015;14:706-14.

26. Cosentyx [package insert]. East Hanover, NJ: Novartis; 2018.

27. Taltz [package insert]. Indianapolis, IN: Eli Lilly and Company; 2020.

28. de La Forest Divonne M, Gottenberg JE, Salliot C. Safety of biologic DMARDs in RA patients in real life: a systematic literature review and meta-analyses of biologic registers. Joint Bone Spine 2017;84:133-40.

29. Dorhoi A, Kaufmann SHE. Tumor necrosis factor alpha in mycobacterial infection. Semin Immunol 2014;26:203-9.

30. Tsai TF, Ho V, Song M, Szapary P, Kato T, Wasfi Y, et al. The safety of ustekinumab treatment in patients with moderate-to-severe psoriasis and latent tuberculosis infection. Br J Dermatol 2012;167:1145-52.

31. Cantini F, Nannini C, Niccoli L, Petrone L, Ippolito G, Goletti D. Risk of tuberculosis reactivation in patients with rheumatoid arthritis, ankylosing spondylitis, and psoriatic arthritis receiving non-anti-TNF-targeted biologics. Mediators Inflamm 2017;2017:8909834.

32. Kammüller M, Tsai TF, Griffiths CEM, Kapoor N, Kolattukudy PE, Brees D, et al. Inhibition of IL-17A by secukinumab shows no evidence of increased Mycobacterium tuberculosis infections. Clin Transl Immunology 2017;6:e152.

33. Fowler E, Ghamrawi RI, Ghiam N, Liao W, Wu JJ. Risk of tuberculosis reactivation during interleukin-17 inhibitor therapy for psoriasis: a systematic review. J Eur Acad Dermatol Venereol 2020;34:1449-56

34. Puig L, Tsai TF, Bhutani T, Uy J, Ramachandran P, Song M, et al. Safety in moderate-to-severe plaque psoriasis patients with latent tuberculosis treated with guselkumab and anti-tuberculosis treatments concomitantly: results from pooled phase 3 VOYAGE 1 and VOYAGE 2 trials. J Eur Acad Dermatol Venereol 2020;34:1744-9.

35. McInnes IB, Mease PJ, Kirkham B, Kavanaugh A, Ritchlin CT, Rahman P, et al. Secukinumab, a human anti-interleukin$17 \mathrm{~A}$ monoclonal antibody, in patients with psoriatic arthritis
(FUTURE 2): a randomised, double-blind, placebo-controlled, phase 3 trial. Lancet 2015;386:1137-46.

36. Mease PJ, van der Heijde D, Ritchlin CT, Okada M, Cuchacovich RS, Shuler CL, et al. Ixekizumab, an interleukin-17A specific monoclonal antibody, for the treatment of biologic-naive patients with active psoriatic arthritis: results from the 24-week randomised, double-blind, placebo-controlled and active (adalimumab)-controlled period of the phase III trial SPIRIT-P1. Ann Rheum Dis 2017;76:79-87.

37. Nash P, Kirkham B, Okada M, Rahman P, Combe B, Burmester GR, et al. Ixekizumab for the treatment of patients with active psoriatic arthritis and an inadequate response to tumour necrosis factor inhibitors: results from the 24-week randomised, double-blind, placebo-controlled period of the SPIRIT-P2 phase 3 trial. Lancet 2017;389:2317-27.

38. Mease P, Roussou E, Burmester GR, Goupille P, Gottlieb A, Moriarty SR, et al. Safety of ixekizumab in patients with psoriatic arthritis: results from a pooled analysis of three clinical trials. Arthritis Care Res 2019;71:367-78.

39. Kavanaugh A, Mease PJ, Reimold AM, Tahir H, Rech J, Hall S, et al. Secukinumab for long-term treatment of psoriatic arthritis: a two-year followup from a phase III, randomized, double-blind placebo-controlled study. Arthritis Care Res 2017;69:347-55.

40. Gordon KB, Blauvelt A, Papp KA, Langley RG, Luger T, Ohtsuki $\mathrm{M}$, et al. Phase 3 trials of ixekizumab in moderate-to-severe plaque psoriasis. N Engl J Med 2016;375:345-56.

41. Griffiths CE, Reich K, Lebwohl M, van de Kerkhof P, Paul C, Menter A, et al. Comparison of ixekizumab with etanercept or placebo in moderate-to-severe psoriasis (UNCOVER-2 and UNCOVER-3): results from two phase 3 randomised trials. Lancet 2015;386:541-51.

42. Strober B, Leonardi C, Papp KA, Mrowietz U, Ohtsuki M, Bissonnette R, et al. Short- and long-term safety outcomes with ixekizumab from 7 clinical trials in psoriasis: etanercept comparisons and integrated data. J Am Acad Dermatol 2017;76:432-40.

43. Langley RG, Elewski BE, Lebwohl M, Reich K, Griffiths CEM, Papp K, et al. Secukinumab in plaque psoriasis--results of two phase 3 trials. N Engl J Med 2014;371:326-38.

44. Miossec P, Korn T, Kuchroo VK. Interleukin-17 and type 17 helper T cells. N Engl J Med 2009;361:888-98.

45. Hueber W, Sands BE, Lewitzky S, Vandemeulebroecke M, Reinisch W, Higgins PDR, et al. Secukinumab, a human anti-IL-17A monoclonal antibody, for moderate to severe Crohn's disease: unexpected results of a randomised, double-blind placebo-controlled trial. Gut 2012;61:1693-700.

46. Targan SR, Feagan B, Vermeire S, Panaccione R, Melmed GY, Landers $\mathrm{C}$, et al. A randomized, double-blind, placebo-controlled phase 2 study of brodalumab in patients with moderate-to-severe Crohn's disease. Am J Gastroenterol 2016;111:1599-607.

47. Mease PJ, Kavanaugh A, Reimold A, Tahir H, Rech J, Hall S, et al. Secukinumab in the treatment of psoriatic arthritis: efficacy and safety results through 3 years from the year 1 extension of the randomised phase III FUTURE 1 trial. RMD Open 2018; $4: \mathrm{e} 000723$.

48. Fieldhouse KA, Ukaibe S, Crowley EL, Khanna R, O’Toole A, Gooderham MJ. Inflammatory bowel disease in patients with psoriasis treated with interleukin-17 inhibitors. Drugs Context 2020;9:2020-2-1.

49. Blauvelt A, Gordon K, Griffiths CEM, Papp KA, Foley P, Song M, et al. Long-term safety of guselkumab: Results from the VOYAGE 1 and VOYAGE 2 trials with up to 5 years of treatment. Poster presented at: American Academy of Dermatology; 2021 April 23-25. 